\title{
A crisis information propagation model based on a competitive relation
}

\author{
$\mathrm{Li} \mathrm{Yang}^{1} \cdot$ Jiaxue Wang ${ }^{1} \cdot$ Chongzhi Gao $^{2} \cdot$ Tong $^{\mathrm{Li}^{2}}$
}

Received: 4 December 2017 / Accepted: 27 February 2018 / Published online: 7 March 2018

c) Springer-Verlag GmbH Germany, part of Springer Nature 2018

\begin{abstract}
In this age of freedom of speech, the increasing popularity of various social networking services is accelerating the propagation of crisis information. Many people express their views in an arbitrary way and spread them continuously in the virtual world of social networking since the entrance threshold for these social networking services is low. In this way, extensive social networking crisis information can be produced and propagated. With the assumption that crisis information propagation is a competing process of real information and false information, we discuss the propagation characteristics of crisis information in different stages and propose a crisis information propagation model based on a competitive relation in this paper. Based on computer simulations of the model, we identify some influencing factors that affect crisis information propagation and provide some corresponding solution strategies.
\end{abstract}

Keywords Social network $\cdot$ Information propagation $\cdot$ Crisis information $\cdot$ Competitive relationship

\section{Introduction}

At present, with the rapid development of social networks, the spread of crisis information such as internet rumors has become one of the hot issues in information technology field. Crisis events influence the public's psychology and behavior, mainly through crisis information propagation. People will attempt to obtain information on the crisis through various channels and express their own opinion as a response (Le et al. 2009). Therefore, identifying the mechanism of crisis information propagation (Memos et al. 2017; Gupta et al. 2016; Yuexin et al. 2014; Wen et al. 2015) and controlling crisis information diffusion effectively (Wu et al. 2016a, b;

Li Yang

yangli@xidian.edu.cn

Jiaxue Wang

1506209190@qq.com

Chongzhi Gao

czgao@gzhu.edu.cn

Tong Li

litongziyi@mail.nankai.edu.cn

1 Xidian University, Xi' an, China

2 Guangzhou University, Guangzhou, China
Segarra and Ribeiro 2014; Newman 2005) are significant issues.

Social networks have become important platforms on which the mass public can share and exchange information, thus satisfying the social needs of users to a great extent. Various social platforms, such as the Sina microblog, Tencent QQ, WeChat, Facebook, and Twitter, contain a large number of users and various information and continuously exchange information. Real positive information in cyberspace will have a positive effect on both individuals and society and promote economic development, social stability and harmony. By contrast, the spread of false negative information will cause great crises in the economy and social security. Therefore, the analysis and study of information propagation in network space are of great importance and practical significance. With the popularization of new media technologies and the widespread use of social media platforms, the dissemination of crisis information is accelerating. For example, Sina Weibo has become a part of everyday life for many people. Crisis messages can be disseminated throughout the network by forwarding and commenting. The message that is disseminated through social media goes well beyond the original incident. The dissemination of public crisis messages can have a direct impact on the trends and outcomes of events and increase the complexity of the government's handling of these crises (Li et al. 2017). 
Crisis events such as natural disasters, public health, and social security are mostly related to the community, which is the subject of public concern. After the public crisis, the release of real information is always dependent on dissemination by the government and authorities through different channels of communication. In the early stages of the crisis, the public is often in a panic due to the lack of information. There is an urgent need for the public to have information about the crisis. This urgent need and panic psychology will lead to large-scale proliferation of false information in the network space. The widespread proliferation of false information poses a huge threat to individuals and society, such as the large-scale "grab salt" events when the "Japan earthquake nuclear leakage" occurred and the mad purchase of disinfectants, salt, and vinegar during the SARS period. Therefore, the analysis and discussion of crisis information dissemination is of great practical significance for the handling of crisis information.

Public crises are occurring with increasing frequency. How best to deal with crises is a major challenge faced by public policymakers. Therefore, on the basis of studies of a model of crisis information dissemination, our paper proposes corresponding strategies to deal with crisis information according to the experimental results.

This paper mainly addresses the following issues:

(1) What is the mechanism of transmission of crisis information?

(2) What are the main factors that affect the dissemination of crisis information?

(3) What measures should be taken to deal with the further spread of crisis information?

By accurately solving these issues, we mainly contribute to the following aspects. First, we divide the process of crisis information dissemination into three stages: the latency period, the outbreak of the spread period, and the recovery period. Simultaneously, we regard the process of propagation of crisis information as a process of competition between real information and false information and conduct modeling analysis and experimental verification of the competition process of real information and false information in different stages. We then discuss the main factors affecting the propagation of crisis information, which include the selection of different channels, the user's subjective attitude and the involvement of government and authorities. Finally, we put forward corresponding solution strategies according to the different characteristics of the crisis information dissemination modes in the three stages.

The rest of the paper is organized as follows. Section 2 presents related work. Section 3 presents the process analysis of crisis information. We then propose a crisis information propagation model based on the competitive relationship in Sect. 4. Section 5 describes the experiments with our model to verify factors influencing crisis information propagation. Finally, conclusions are presented in Sect. 6.

\section{Related work}

In related work, researchers have performed extensive analyses of information propagation in social networks. Previous studies of crisis information have mainly focused on its dissemination characteristics (Ping et al. 2017; Ibtihal et al. 2017; Alsmirat et al. 2017; Chen and Sakamoto 2014), the identification method ( $\mathrm{Li}$ et al. 2018; Canini et al. 2011; Chen et al. 2010) and response measures(Gupta et al. 2016; Stergiou et al. 2018; Wen et al. 2015; Hyman and $\mathrm{Li}$ 2005). Many researchers have studied the dissemination model(Wu et al. 2016a, b; Lee et al. 2017) of crisis information according to the dissemination characteristics of crisis information. These studies have contributed greatly to research on the dissemination model of crisis information.

Most information transmission models in social networks are transformed from epidemic models (Hyman and Li 2005). Classic models of basic epidemic models (Kim et al. 2006) include SI, SIR, SIRS, and SEIR. The spread of information in social networks is similar to that of infectious diseases. The description of the crisis information diffusion model mainly includes the BASS model (Jiuchang et al. 2011) and Lotka-Volterra model (Austin et al. 2012).

The BASS model is often used to predict the proliferation of new products, new technologies and new concepts in the marketplace. The BASS model treats the dissemination of crisis information as a process of spreading new products in the marketplace. In the process of information diffusion, most users in the network do not know the crisis information, except for a few people who have access to the crisis information. Those who do not know the crisis information are called the potential population of crisis information dissemination.

The Lotka-Volterra diffusion model is a competition model of different species proposed by Lotka and Volterra that is used to describe the process of competition among different species. Lotka and Volterra (Austin et al. 2012) proposed that after the outbreak of a crisis, the diffusion of crisis information is the process of competition between real information and false information.

Research on crisis information mainly focuses on two aspects. One is the analysis of and research on crisis information propagation models; the other is coping strategies for crisis information propagation. Le et al. 
(2009) analyzed the diffusion process of real information and false information in the process of crisis information propagation and divided the process of crisis information spreading after a paroxysmal public crisis breaks out into three periods. Le et al. also discussed the dynamic rules of crisis information spreading on social networks in different stages and proposed a disease infection-like two-stage dynamic crisis information-spreading model. Austin et al. (2012) conducted a detailed analysis of the driving force of information searches and the subjective attitudes of different communication channels after the crisis. Jiuchang et al. (2011) proposed the BASS-based crisis information diffusion model by analyzing the diffusion characteristics of crisis information under different transmission modes. According to the network space change of real information and false information in the process of crisis information transmission, Qi et al. (2012) proposed a diffusion information model based on Lotka-Volterra. Zhixue and Linlin (2009) analyzed the simultaneous spread of positive and negative information from the human-related aspect and proposed an analytical model based on epidemic models. The ICM and the Linear Threshold Model (LTM) (Rahimkhani et al. 2015)are the primary models for information propagation. According to the analysis of the data characteristics of crisis information dissemination, Yuexin et al. (2014) put forward three types of forecasting methods, mathematical prediction, empirical prediction and comprehensive forecasting, to provide a reference for the response to crisis events.

These significant streams in the literature support the development of a better understanding of crisis information propagation. Our paper differs from existing studies in three ways. (1) Based on the Lotka-Volterra diffusion model (Austin et al. 2012), we treat the diffusion model of crisis information as a process of competition between real information and false information and propose a competition model. (2) We discuss three major factors affecting the dissemination of information on crises and achieve good results. (3) According to the influencing factors of crisis information dissemination, we put forward corresponding coping strategies to minimize the impact of crisis information.

\section{Process analysis of crisis information}

\subsection{Communication channel selection}

There are two means of propagating crisis information: traditional media and social network media. The choice of propagation has a certain impact on the real feasibility of crisis information.

Traditional media spreads information to the masses by means of unidirectional broadcasting. Although we cannot obtain feedback, this information is highly credible because of the official authority of the source of information. Thus, the information disseminated through traditional media can generally be identified as real information.

The information propagated through social media is often spread from one node to another through inter-node interconnections. Because the social network itself is a virtual world, each user node can become a communicator to transmit information. In addition, the information release threshold is low, and speech is relatively free in modern society. Consequently, the source of the information has a certain degree of distortion, and the credibility of the information is not high.

\subsection{Statistical analysis}

A crisis often happens suddenly, extensively and continuously. In addition, a crisis can cause a large-scale economic and social crisis or natural disasters, etc. Due to the complexity of the social network itself, the masses respond differently to a crisis. Taking the "Japan Earthquake" as an example, Chen et al. (2014) divided the crisis event into three types: (1) people are located in the earthquake area; (2) people's friends are experiencing earthquakes; (3) neither people themselves nor their friends are in the earthquake area. In addition, he analyzes in detail different reactions to the crisis of people located at different distances from the earthquake region. Wen et al. (2014) analyzed the inner feelings of the masses about rumors and different ways of choosing information. To understand the reasons that rumors go abroad after crisis events, Sina net performed the following

\begin{tabular}{ll}
\hline The factors of crisis information propagation & Proportion (\%) \\
\hline We prefer to believe them rather than ignore them & 32.80 \\
Some people do not have basic scientific literacy and lack self-discrimination & 31.60 \\
Some people deliberately create and spread rumors for individual interests & 21.50 \\
Microblogging, mobile phones, QQ, MSN and other instant communication tools are & 9.80 \\
widely used & \\
Else & 4.20 \\
\hline
\end{tabular}


survey. Taking the "Japan earthquake" as an example, the data from this survey are shown in Table 1.

The survey results reveal that real information and false information constantly impact the public since information is opaque and closely related to the public. However, the masses prefer to believe crisis information rather than ignore it, which promotes the spread of false information and results in immeasurable loss.

\subsection{Four-stage model of crisis information}

The popularity of the network provides a good basis for propagating crisis information. The most classic model of all crisis information propagation models is the four-stage model proposed by Steven Fink.

\section{(1) Latency period}

The latency period is the early stage of crisis information propagation. This stage occurs soon after the crisis event. At this stage, the real information is in the hands of only the few people who have experienced the crisis and has not propagated to the public yet. In addition, many people are in shock induced by the crisis event and have not yet produced a demand for crisis information. Consequently, false information has not yet been born and propagated. From the perspective of crisis information propagation, in this stage, crisis information in the network is basically in a vacuum; the information exists only in a few nodes and has not yet begun to spread. This stage is generally very short. The public who experienced the shock of the first crisis outbreak begins to place greater demands for crisis information, and people attempt to obtain information through various channels. Hence, to obtain information, all types of true and false information begin to propagate in the network. From the perspective of crisis information control, this is the easiest time to deal with crisis information but is also the most difficult period to identify. At this stage, the spread of crisis information is limited and does not greatly impact the network.

(2) Outbreak stage

The influence of crisis information propagation emerges in the outbreak stage. After entering this stage, the public is eager to understand the causes of and latest developments in the crisis, and the demand for crisis information sharply increases. This period is short, and the group of people will be affected by both real information and false information. As a result, people have doubts about the authenticity of information, which greatly impacts the public psychology. Crisis information begins to spread. Initially, only a few nodes grasp the true message of the crisis, and it takes time for the true information from these nodes to spread abroad to the network. Thus, for a considerable period of time, only those nodes around the initial nodes can receive real information on the crisis. To meet the public demand for information about the crisis, false information begins to spread to the other nodes in the network at a very fast speed. At this stage, extensive false information floods the network and mixes with real information. Simultaneously, the major spreading media are individual and some non-authoritative media, and the information identification capacity of the general public is limited. Even if some nodes have the opportunity to receive true information, the subsequent impact of the abundant false information may disrupt the formation of the correct perspective and change these nodes into rumor spreaders.

(3) Spread stage

Crisis information spreads in the network in this stage. This is the longest of the four phases and is a process of real information and false information competing with each other in the network.

(4) Recovery stage

The crisis is resolved in this stage. The government and authoritative organizations are involved in handling crisis information during this time. They devote themselves to clarifying and dealing with false information.

The four-stage model of crisis information propagation is a good tool for revealing the law of crisis information transmission and provides a basis for studying crisis information propagation. Researchers have also performed relevant research on the crisis information propagation model. Le et al. (2009) analyzed the characteristics of crisis information transmission in different periods and proposed a twostage dynamic model of crisis information dissemination on social networks. Austin et al. (2012) studied the impact of different transmission pathways on crisis information dissemination.

\section{The crisis information propagation model}

After crisis events, there are two types of information: real information and false information. Crisis information propagation is a competing process of real information and false information. In this section, we mainly analyze the mutual competition between the two types of information in the process of crisis information transmission and discuss the propagation characteristics of crisis information in different stages. 


\subsection{Crisis information propagation characteristics}

According to the four-stage model of crisis information propagation, the outbreak stage and spread stage can be merged into a single stage. In this stage, the statuses of users can be divided into three categories:

(1) Unknown: individuals who have not heard any information.

(2) Truth: those who are communicating the real information.

(3) Faker: those who are communicating false information.

The changes in the statuses of users during crisis information transmission are shown in Fig. 1.

For convenience, we list the major variables used below and explain them in Table 2.

In the crisis outbreak and spread stage, the government and authorities are not involved, and thus, Faker becomes

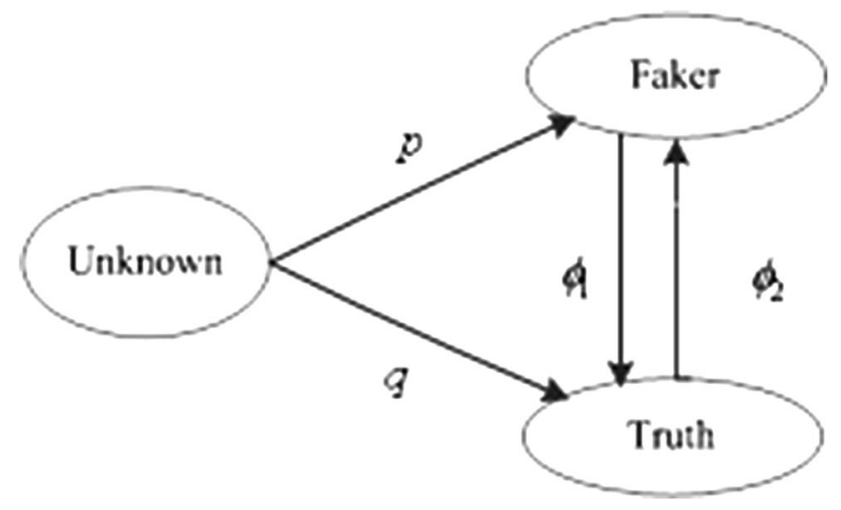

Fig. 1 Changes in the statuses of users during crisis information transmission
Truth with probability $\phi_{2}$. In this stage, due to the opacity of information, Faker may again become Truth with probability $\phi_{1}$. In the information recovery stage, the government and the authorities begin to intervene. They clarify the false information and solve other public matters. At this point, Faker becomes Truth with probability $\phi_{1}$, but Truth no longer becomes Faker. In this period, $\phi_{2}$ is equal to 0 .

Assume that the size of the network space is $\mathrm{N}$. We introduce $\mathbf{S}(\mathbf{t})^{\mathbf{U}}$ to denote the size of the network space of unknown at time t. The symbols $\mathbf{S}(\mathbf{t})^{\mathbf{F}}$ and $\mathbf{S}(\mathbf{t})^{\mathbf{T}}$ are used to denote the sizes of the network spaces of Faker and Truth, respectively. We can obtain the size of the network space of unknown in (1):

$S(t)^{U}=N-S(t)^{F}-S(t)^{T}$

The symbol $\mathrm{p}$ is used to denote the probability that the user will change from unknown to Faker. We use q to denote the probability that the user will change from unknown to Truth. The size of the network space of Truth is shown as (2):

$S(t+1)^{T}=S(t)^{T}+S(t)^{U} * q-S(t)^{T} * \phi_{2}+S(t)^{F} * \phi_{1}$

The size of the network space of Faker is defined by (3):

$S(t+1)^{F}=S(t)^{T}+S(t)^{U} * p+S(t)^{T} * \phi_{2}-S(t)^{F} * \phi_{1}$

As a result, users in each state occupying the size of the network space are given by (4):

$$
\begin{aligned}
S(t)^{U} & =N-S(t)^{F}-S(t)^{T} \\
S(t+1)^{T} & =S(t)^{T}+S(t)^{U} * q-S(t)^{T} * \phi_{2}+S(t)^{F} * \phi_{1} \\
S(t+1)^{F} & =S(t)^{T}+S(t)^{U} * p+S(t)^{T} * \phi_{2}-S(t)^{F} * \phi_{1}
\end{aligned}
$$

During the crisis, the number of people who prefer the real information in cyberspace is time-varying. As time goes on,
Table 2 Explanation of the major symbols

\begin{tabular}{ll}
\hline Symbol & Explanation \\
\hline $\mathbf{S}(\mathbf{t})^{\mathbf{U}}$ & The size of the network space of unknown at time t \\
$\mathbf{S}(\mathbf{t})^{\mathbf{T}}$ & The size of the network space of Truth at time t \\
$\mathbf{S}(\mathbf{t})^{\mathbf{F}}$ & The size of the network space of Faker at time t \\
$\lambda_{1}$ & The probability of choosing traditional media as a communication channel \\
$\lambda_{2}$ & The probability of choosing social networks as a communication channel \\
$\eta^{\mathbf{T}}$ & The intervention time of the government and the authorities in the crisis event \\
Unknown & Individuals have not heard any information \\
Truth & Communicators of real information \\
Faker & Communicators of false information \\
$\mathrm{p}$ & The probability of unknown becoming Faker \\
$\mathrm{q}$ & The probability of unknown becoming Truth \\
$\mathrm{N}$ & The size of the network space \\
$\phi_{1}$ & The probability of Faker becoming Truth \\
$\boldsymbol{\phi}_{2}$ & The probability of Truth becoming Faker \\
\hline
\end{tabular}


the government or the authorities intervene in the crisis and posts real information quickly to clarify false information. Due to the authenticity and authority of the government, some people begin to trust the real information.

\subsection{Communication channel selection}

After the crisis outbreak, information propagation mainly includes two channels. They are as follows.

(1) Traditional media At the beginning of the crisis, transmitters spread the information to the masses by means of television, radio, newspaper, etc. The transmission is unidirectional. Traditional social media are often identified by real-name systems. Governments and authorities disseminate information through traditional media, and thus, the authenticity of the information sources is considerable. We use $\lambda_{1}$ to denote the probability of choosing the traditional media as a communication mode. The trust value of traditional media is very high, and thus, we set the authenticity of the information to 1.

(2) Social network A node propagates information to his neighbor nodes at a certain probability in the social network. However, due to the low restrictions on the use of social networks, the network contains various information from all types of people. In order to win the attention of users, publishers may spread exaggerated, fuzzy or mysterious information that often deviates from fact through the baseless assertion of the network users. Therefore, information disseminated through social networks has a certain degree of distortion. The symbol $\lambda_{2}$ is used to denote the probability of choosing social networks as a communication channel. In the different stages of crisis information transmission, the competition between real information and false information is shown in Fig. 2.

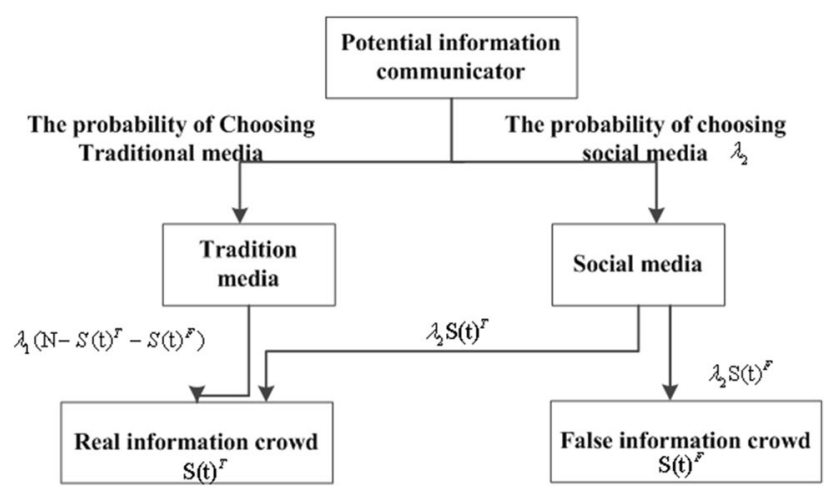

Fig. 2 Crisis information propagation via different communication channels
- During the crisis event, to ensure the authenticity of released information, the government and other authorities rarely issue crisis-related information via traditional media. However, users in social networks have a lower sense of responsibility to ensure the authenticity of information, which will frequently lead to filling of the network with various types of crisis information. The abundance of this information increases as time goes on. When users want to know more about the crisis, they may choose both traditional media and social media. Thus, the sum of $\lambda_{1}$ and $\lambda_{2}$ may be greater than or equal to 1 ; that is, $\lambda_{1}+\lambda_{2} \geq 1$. After the crisis outbreak, the size of the real information in the network space is defined as the sum of the real information spread through traditional media and social media in the network, which is expressed by (5):

$S(t+1)^{T}=\lambda_{1}\left(N-S(t)^{T}-S(t)^{F}\right)+\lambda_{2} S(t)^{T}$

- After the crisis outbreak, it is assumed that all real information comes from traditional media and all false information come from social networks. Thus, the size of the false information network space is expressed by (6):

To realize the construction of the crisis information prop$S(t+1)^{F}=\lambda_{2} S(t)^{F}$

agation model, the following measures should be considered. Taking the intervention time $\boldsymbol{\eta}^{\mathbf{T}}$ of the government and the authorities in this crisis event as a boundary, the spread of crisis information is divided into two stages: the crisis outbreak stage and crisis recovery stage. We analyze the changes in the size of users in the network space in different stages. We also analyze the process of crisis information spreading in different communication channels. As a result, we obtain the change capacity of the network space, in which real information and false information are spread through two communication channels.

In order to describe the selection process of communication channels in the dissemination of crisis information, we propose Algorithm 1. At the very beginning of a crisis event, the cyberspace is $\mathrm{N}$ and the values of $\mathbf{S}(\mathbf{t})^{\mathbf{T}}$ and $\mathbf{S}(\mathbf{t})^{\mathbf{F}}$ are zero. With the spread of crisis information, the crisis information in cyberspace is constantly increasing. Thus, the values of $\mathbf{S}(\mathbf{t})^{\mathbf{T}}$ and $\mathbf{S}(\mathbf{t})^{\mathbf{F}}$ continue to increase and compete with each other. As shown in Fig. 2, crisis information is disseminated through traditional media and social networks with a certain probability.

In Algorithm 1, we can obtain the changes in the crisis information network space size from the output, and after several iterations, the network space size of crisis information will be stable. We set different values for parameters $\lambda_{1}$ and $\lambda_{2}$ to analyze the impact of different communication 
channels on the size of the network space occupied by crisis information.

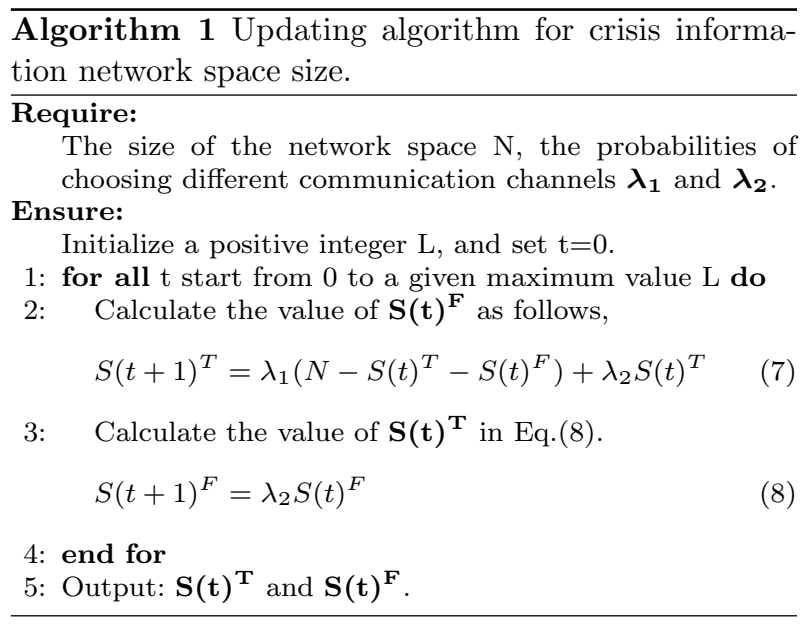

In addition, given the size of the network space $\mathrm{N}$, we can analyze the impact of different communication channels on the size of the network space occupied by crisis information by changing the values of $\lambda_{1}$ and $\lambda_{2}$. The results are shown in Sect. 5.

From algorithm 1, we known that the time complexity of the algorithm is only related to the value of $\mathrm{L}$. Therefore, the time complexity of our algorithm is $\mathrm{O}(\mathrm{L})$. The algorithm updates two values at each iteration, and thus, the spatial complexity is related to the number of iterations. As a result, the spatial complexity is $\mathrm{O}(\mathrm{L})$. The algorithm can be applied in the general network.

\section{The experiment}

To study the factors impacting crisis information propagation on the social network, we simulate the propagation model based on the competitive relationship on a computer. Based on the crisis information propagation model established in Sect. 4, we analyze and discuss the three influencing factors of crisis information propagation in this section. The influencing factors include the probability of choosing different communication channels, the group pessimistic attitude of the public, and the time of government and authority intervention.

The dataset was collected from the Sina microblog, one of the most commonly used and largest social networks in China, from November 2015 to January 2016. From the collection, we obtain 38,225 user nodes. Considering the fact that the size of the network does not affect the crisis information propagation process, we set up a small world network that contains 1000 nodes in the computer.

For convenience, we list the major factors influencing the propagation of crisis information and the corresponding impacts in Table 3. The following experiment will verify the correctness of these factors.

\subsection{The impact of the selection of different channels}

Crisis information communication channels mainly include two types: traditional media and social media. After the outbreak of crisis information, crisis events are closely related to the public, which stimulates the mass requirement for crisis information. In traditional media, most of the communicators of information are governments and authorities, and thus, all of the released information is considered true information. By contrast, in social media, real information and false information are mixed together. Studies have been performed to better understand the impact of the selection of different channels on the spread of crisis information. Zhang et al. (2009) experimentally verified that the size of the network does not affect the crisis information propagation process. Therefore, we build a small network that contains 1000 nodes. By analyzing the propagation model in different stages of crisis information transmission, we elucidate the impact of different communication channels on the size of the network space occupied by crisis information. These impacts can be seen in Fig. 3 .

In Fig. 3, the abscissa is the time of crisis information dissemination and the ordinate is the value of the network space occupied by the crisis information. This value reflects the size of the spread of real and false information in cyberspace. As shown in Fig. 3, the larger the value of $\lambda$, the faster the size of the network space occupied by crisis information increases, as demonstrated by comparing the two curves $\lambda_{1}+\lambda_{2}=0.4+0.4$ and $\lambda_{1}+\lambda_{2}=0.6+0.6$. In other words, the greater the probability of choosing traditional

Table 3 The influencing factors and their impacts on crisis information propagation

\begin{tabular}{lll}
\hline Factors & Is there any impact? & Impacts \\
\hline Different channel selection & Yes & The greater the value of $\boldsymbol{\lambda}_{\mathbf{2}}$, the faster the dissemination of crisis information \\
User's subjective attitude & Yes & The user's pessimistic attitude can speed up the dissemination of crisis information \\
Involvement & Yes & The smaller the value of $\boldsymbol{\eta}^{\mathbf{T}}$, the less false information in cyberspace \\
\hline
\end{tabular}




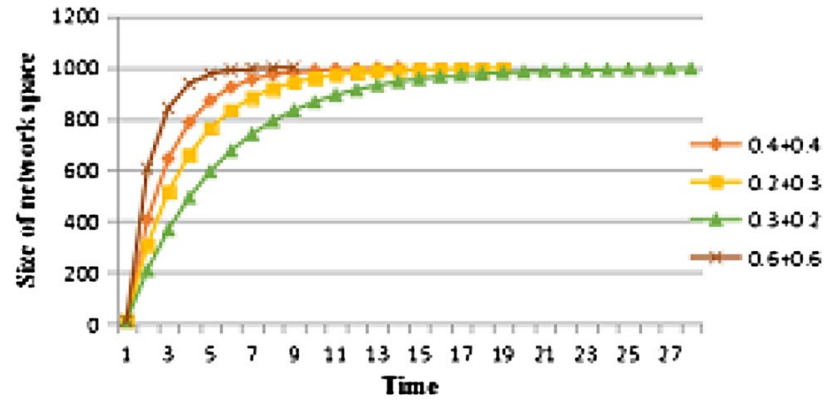

Fig. 3 Changes in the size of the network space with the growth of $\lambda_{1}$ and $\lambda_{2}$

media and social media, the faster the dissemination of crisis information. Therefore, we can draw the conclusion that the dissemination of crisis information is related to the choice of transmission channels. Similarly, the larger the value of $\lambda_{2}$, the faster the size of the network space occupied by crisis information increases, as demonstrated by comparing the two curves $\lambda_{1}+\lambda_{2}=0.2+0.3$ and $\lambda_{1}+\lambda_{2}=0.3+0.2$. The same result can be obtained by comparing any other arbitrary curves.

In conclusion, we can conclude that the dissemination of crisis information is related to the selection of its transmission channel. The greater the probability of choosing social media, the faster the dissemination of crisis information.

\subsection{The influence of the user's subjective attitude}

After the outbreak of the crisis, users in the network are often pessimistic about the information on the crisis before the government and the authorities have dealt with the crisis. As shown in Table 1, there are $64.4 \%$ pessimistic users in the network. In the outbreak of crisis information, when the false information and real information continue to psychologically impact users, the user state will change from the Faker state to the Truth state with a certain probability or may change from the Truth state to the Faker state. The probability that the user state transits from the Faker state to the Truth state is $\phi_{1}$, and the probability that the user state changes from the Truth state to the Faker state is $\phi_{2}$.

Crisis information propagation is often closely related to people. As shown in Table 1, users often adopt a pessimistic attitude toward the crisis. After the crisis, if the government and authorities do not perform timely public relations processing, the network will be filled with real information and false information. At this point, most users will adopt a pessimistic attitude, which will impact the spread of crisis information. We introduce $\mathrm{T}$ to denote changes in the network space occupied by Truth. $\mathrm{F}$ and $\mathrm{U}$ represent changes in the network space occupied by Faker and unknown, respectively. We can obtain the results from Figs. 4 and 5.

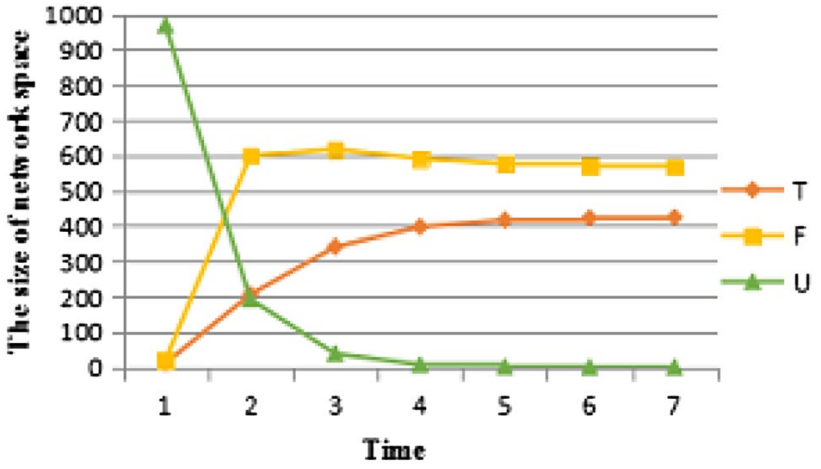

Fig. 4 The change in the network space state in the crisis outbreak stage with $\mathrm{p}=0.2$

In Figs. 4 and 5, the abscissa is time, and the ordinate is the size of the network space. We introduce $p$ to denote the probability of a pessimistic user attitude about the crisis. As shown in Fig. 4, the cyberspace tends to stabilize at approximately 4 time units when $\mathrm{p}=0.2$. According to Fig. 5, the cyberspace tends to stabilize at approximately 11 time units when $p=0.6$. Therefore, Figs. 4 and 5 reveal that the larger the value of $p$, the shorter the time required for the cyberspace to stabilize. In other words, in the outbreak of crisis information, users in cyberspace tend to be pessimistic about crisis information due to the absence of intervention by government and authorities, resulting in the rapid spread of crisis information in cyberspace.

\subsection{Involvement of government and authorities}

The occurrence of a crisis is closely related to national and social security and is inseparable from the life of the masses. The speculation and commentary of general users on crisis events are often questioned, and the masses are most vulnerable to the involvement of the government and authorities. The government's clarification represents the optimal suppression of the spread of false information. The influence

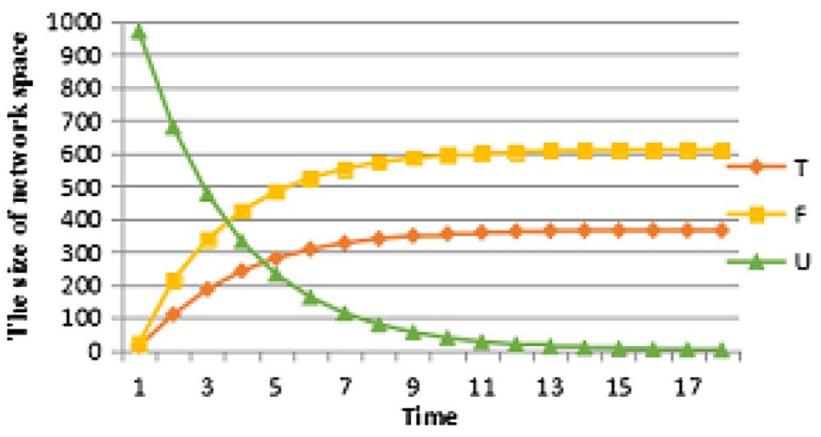

Fig. 5 The change in the network space state in the crisis outbreak stage with $p=0.6$ 


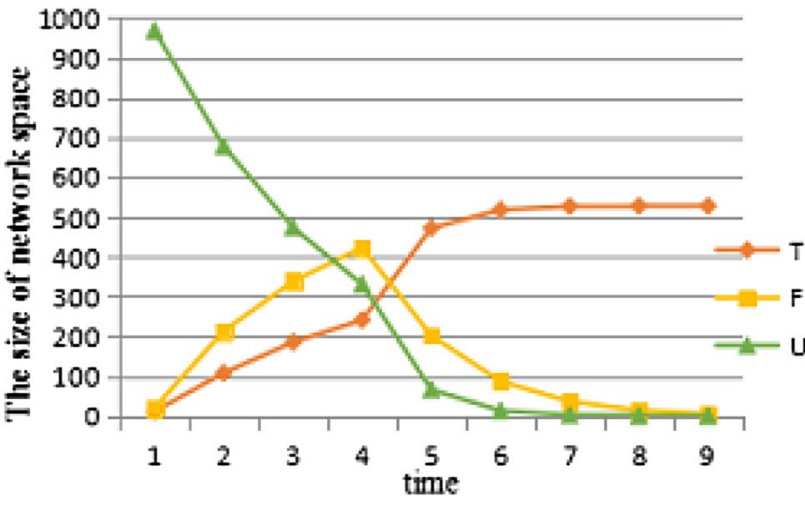

Fig. 6 Changes in the crisis information network space with $\eta^{\mathbf{T}}=4$

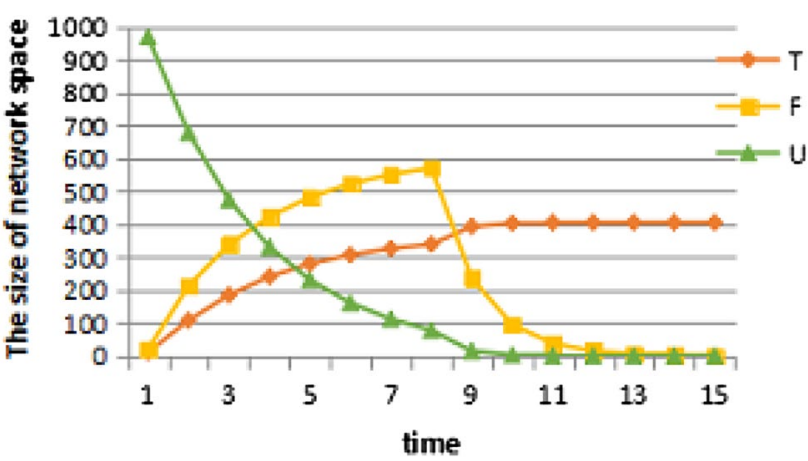

Fig. 7 Changes in the crisis information network space with $\eta^{T}=6$

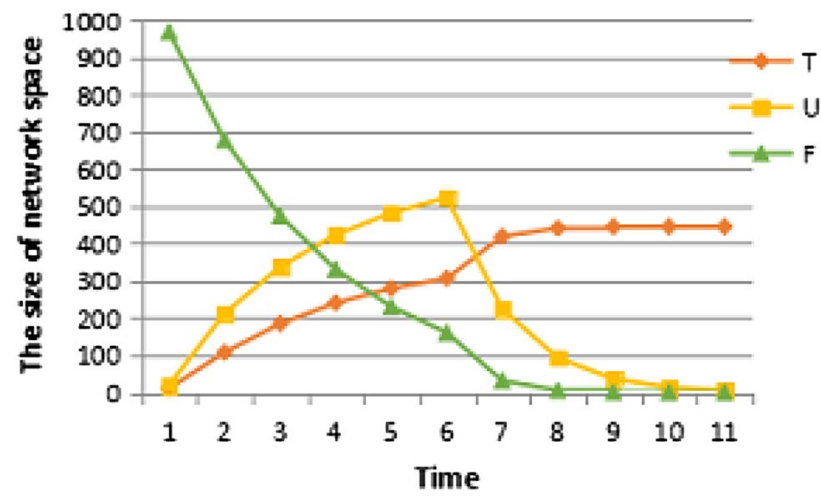

Fig. 8 Changes in the crisis information network space with $\eta^{T}=8$

of the government and authorities on the spread of crisis information at different times is shown in Figs. 6, 7 and 8.

In Figs. 6, 7, and 8, the abscissa is time, and the ordinate is the size of the network space. The symbol $\boldsymbol{\eta}^{\mathbf{T}}$ is used to denote the intervention time of the government and the authorities in this crisis event. As shown in Fig. 6, cyberspace tends to stabilize at approximately 8 time units when $\boldsymbol{\eta}^{\mathbf{T}}=4$. In Fig. 7, cyberspace tends to stabilize at approximately 10 time units when $\eta^{T}=6$. As shown in

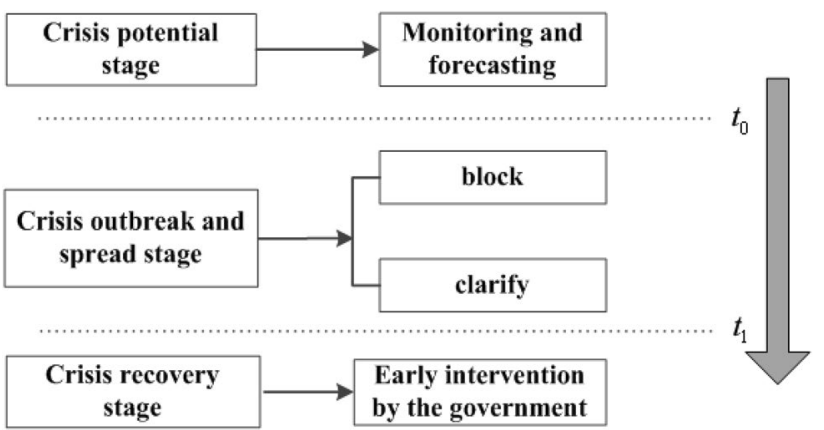

Fig. 9 Crisis information response measures in different stages

Fig. 8, cyberspace tends to stabilize at approximately 12 time units when $\boldsymbol{\eta}^{\mathbf{T}}=8$. By comparing Figs. 6, 7 and 8, we can see that the larger the value of $\boldsymbol{\eta}^{\mathbf{T}}$, the shorter the time required for cyberspace stabilization. In other words, the sooner the government and the authorities intervene, the smaller the spread of the maximum value of false information among crisis information. Simultaneously, real information grows rapidly in cyberspace. Thus, the government and the authorities should be involved as soon as possible in crisis information processing to achieve better results.

\subsection{Responses in different stages}

Based on the analysis and discussion of the influencing factors of crisis information, we put forward corresponding countermeasures according to the characteristics of information propagation at different stages. The countermeasures are shown in Fig. 9.

In Fig. 9, $\mathbf{t}_{\mathbf{0}}$ indicates the time at which the crisis broke out. Based on the above analysis and discussion, we observe that before time $\mathbf{t}_{\mathbf{0}}$, the crisis information is at the potential stage. The impact of this time does not spread. Therefore, this stage is the best time for crisis information processing. We can forecast and monitor crisis information in a timely manner to prevent the outbreak of crisis information. $\mathbf{t}_{\mathbf{1}}$ indicates the time at which the government and the authorities intervene. Based on the above discussion and analysis, the earlier the time of government intervention, the greater the effectiveness of crisis information dissemination, and the smaller the impact of the crisis information.

(1) During the latency period, crisis information is only in the hands of a small number of people. The public does not realize the occurrence of the crisis and the possible consequences. The crisis does not cause public panic and has not aroused the group's urgent need for crisis information. Crisis information has not yet spread to 
key hub nodes in the network. This stage is the best time for crisis information processing.

(2) After the outbreak of the crisis, crisis information processing often contains two aspects: the suppression of false information and the publication of real information. Based on the analysis and discussion of the influencing factors of crisis information, we can recommend the following measures.

- The key nodes in the network are often the only way to maximize the diffusion of crisis information. We should monitor the key nodes in the network and block the critical nodes that spread false information in a timely manner to suppress the flow of false information at critical nodes.

- It is important to accelerate the spread of real information. The greater the probability of selection, the greater the value of real information network space in the network. Therefore, we should strengthen the dissemination of real information by television, newspapers, radio and other traditional media. We can strengthen traditional media and social media links through the establishment of official social accounts in the social network, etc. In addition, we should establish a timely crisis information release mechanism.

- User pessimism about crisis information tends to contribute to the further spread of false information. Therefore, we should maintain a scientific and active attitude. Public official mainstream media with high credibility should be our choice for information channels.

\section{Conclusion}

Based on research on the spread of crisis information of concern to all people, this article analyzes the different stages of crisis information dissemination. Based on simulation experiments, we conducted research on different influencing factors, including the selection of different communication channels, users' subjective attitudes, and the involvement of the government and authorities, and proposed coping strategies.

After the outbreak of a crisis, crisis information spreads through various channels. If we do not deal with the crisis information in time, a more serious secondary crisis may occur. Based on the statistics of crisis information propagation factors, this paper analyzes and studies the propagation characteristics of crisis information at different propagation stages and obtains the following conclusions: (1) the greater the probability of choosing traditional media and social media is, the faster the growth rate of the network space occupied by crisis information; (2) a pessimistic attitude of the mass public toward crisis information will cause the further spread of false information; and (3) the timeliness of the handling of the crisis by the government and the authorities affects the size of the network space occupied by both real information and false information.

With respect to the study of the communication characteristics of crisis information, this paper only analyzes and discusses from the perspective of mathematical analysis, which has some defects. Additionally, the discussion of the influencing factors of crisis information dissemination is not sufficiently comprehensive and requires further exploration. In the future, we will conduct the following research:

(1) Although this paper analyzes and verifies the influencing factors that affect crisis information dissemination and achieves good results, the factors have different weights in the whole process of crisis information dissemination and different impacts on crisis information dissemination. Therefore, to minimize the impact of crisis information, further research on the weights of influencing factors of crisis information is of practical significance and urgently needed.

(2) Research on crisis information dissemination has become a hot issue. This paper discussed the propagation of crisis information via a series of mathematical analyses. The potential stage is the best period to deal with crisis information. The prediction of crisis information propagation in the potential period is of great practical significance to handle crisis events and requires further in-depth study.

Acknowledgements We would like to thanks the anonymous reviewers for their careful reading and useful comments. This work was supported by the National Natural Science Foundation of China (61671360, 61672415), the Key Program of NSFC-Tongyong Union Foundation under Grant (U1636209), the Key Program of NSFC Grant (U1405255), the Natural Science Basic Research Plan in Shanxi Province of China (2017JM6082) and the China 111 Project (B16037).

\section{References}

Alsmirat MA, Jararweh Y, Obaidat I, Gupta BB (2017) Internet of surveillance: a cloud supported large-scale wireless surveillance system. J Supercomput 73(3):1-20. https://doi.org/10.1007/s1122 7-016-1857-x

Austin L, Liu BF, Jin Y (2012) How audiences seek out crisis information: exploring the social-mediated crisis communication model. J Appl Commun Res. https://doi.org/10.1080/00909 882.2012.654498

Canini KR, Suh B, Pirolli PL (2011) Finding credible information sources in social networks based on content and social structure. DBLP. https://doi.org/10.1109/PASSAT/SocialCom.2011.91

Chen W, Yuan Y, Zhang L (2010) Scalable influence maximization in social networks under the linear threshold model. In: IEEE 
International Conference on Data Mining. IEEE Computer Society, pp 88-97. https://doi.org/10.1109/ICDM.2010.118

Chen R, Sakamoto Y (2014) Feelings and perspective matter: sharing of crisis information in social media. In: Hawaii International Conference on System Sciences. IEEE, pp 1958-1967. https://doi. org/10.1109/HICSS.2014.248

Granovetter M (1973) The strength of weak ties. Am J Sociol. https:// doi.org/10.1016/B978-0-12-442450-0.50025-0

Gupta S, Gupta BB (2016) XSS-secure as a service for the platforms of online social network-based multimedia web applications in cloud. Multimed Tools Appl. https://doi.org/10.1007/s11042-016-3735-1

Gupta BB, Agrawal DP, Yamaguchi S (2016) Handbook of research on modern cryptographic solutions for computer and cyber security. IGI Global. https://doi.org/10.4018/978-1-5225-0105-3

Hosseini S, Azgomi MA (2016) A model for malware propagation in scale-free networks based on rumor spreading process. Comput Netw 108(C):97-107. https://doi.org/10.1016/j.comne t.2016.08.010

Hyman J, Li J (2005) Differential susceptibility epidemic models. J Math Biol 50(6):626-644. https://doi.org/10.1007/s0028 5-004-0301-7

Ibtihal M, Driss EO, Hassan N (2017) Homomorphic encryption as a service for outsourced images in mobile cloud computing environment. Int J Cloud Appl Comput 7(2):27-40. https://doi. org/10.4018/IJCAC.2017040103

Jiuchang W, Lei Z, Dingtao Z (2011) Crisis information diffusion model based on BASS model. Syst Eng 16-22

Kim J, Radhakrishnan S, Jang J (2006) Cost optimization in SIS model of worm infection. ETRI J 28(5):692-695. https://doi. org/10.4218/etrij.06.0206.0026

Le Z, Qi Z, Wei Q (2009) Two-stage dynamics of crisis information dissemination on social network. In: International Conference on Management Science and Engineering. IEEE, pp 2117-2122. https://doi.org/10.1109/ICMSE.2009.5317652

Lee K, Choi HO, Min SD, Lee J, Gupta BB (2017) A comparative evaluation of atrial fibrillation detection methods in koreans based on optical recordings using a smartphone. IEEE Access 99:1-1. https://doi.org/10.1109/ACCESS.2017.2700488

Li Y, Yaping T, Jianfeng M (2017) Identifying opinion leaders in social networks with topic limitation. Cluster Comput. https:// doi.org/10.1007/s10586-017-0732-8

Li Y, Yafeng Q, Zhihong L, Jianfeng M, Xinhua L (2018) Identifying opinion leader nodes in online social networks with a new closeness evaluation algorithm. Soft Comput 22(2):453-464. https:// doi.org/10.1007/s00500-016-2335-3

Memos VA, Psannis KE, Ishibashi Y, Kim BG, Gupta BB (2017) An efficient algorithm for media-based surveillance system (EAM$\mathrm{SuS}$ ) in IoT smart city framework. Future Gener Comput Syst. https://doi.org/10.1016/j.future.2017.04.039
Newman MEJ (2005) A measure of betweenness centrality based on random walks. Soc Netw. https://doi.org/10.1016/j.socne t.2004.11.009

Pei S, Muchnik L Jr, AJ (2013) Searching for superspreaders of information in real-world social media. Sci Rep 4:5547. https://doi. org/10.1038/srep05547

Ping L, Jin L, Zhengan H, Tong L, Chongzhi G, Siuming Y, Kai C (2017) Multi-key privacy-preserving deep learning in cloud computing. Future Gener Comput Syst. https://doi.org/10.1016/j.futur e.2017.02.006

Qi Z, Wei Q, Le Z (2012) Social-pattern crisis information diffusion model under Lotka-Volterra system. Syst Eng Theory Pract. https ://doi.org/10.3969/j.issn.1000-6788.2012.01.013

Rahimkhani K, Aleahmad A, Rahgozar M, Moeini A (2015) A fast algorithm for finding most influential people based on the linear threshold model. Expert Syst Appl Int J 42(3):1353-1361. https ://doi.org/10.1016/j.eswa.2014.09.037

Segarra S, Ribeiro A (2014) A stable betweenness centrality measure in networks. In: IEEE International Conference on Acoustics, Speech and Signal Processing. IEEE, pp 3859-3863. https://doi. org/10.1109/ICASSP.2014.6854324

Stergiou C, Psannis KE, Kim BG, Gupta B (2018) Secure integration of IoT and Cloud Computing. Future Gener Comput Syst. https:// doi.org/10.1016/j.future.2016.11.031

Wen S, Jiang J, Xiang Y (2014) To shut them up or to clarify: restraining the spread of rumors in online social networks. Parallel Distrib Syst. https://doi.org/10.1109/TPDS.2013.2297115

Wen S, Haghighi MS, Chen C (2015) A sword with two edges: propagation studies on both positive and negative information in online social networks. IEEE Trans Comput. https://doi.org/10.1109/ TC.2013.2295802

Wu J, Guo S, Li J, Zeng D (2016) Big data meet green challenges: big data toward green applications. IEEE Syst J 10(3):888-900. https ://doi.org/10.1109/JSYST.2016.2550530

Wu J, Bisio I, Gniady C, Hossain E (2014) Context-aware networking and communications: Part 1. IEEE Commun Mag 52(8):64-65. https://doi.org/10.1109/MCOM.2014.6871671

Wu J, Guo S, Li J, Zeng D (2016) Big data meet green challenges: greening big data. IEEE Syst J 10(3):873-887. https://doi. org/10.1109/JSYST.2016.2550538

Yuexin L, Xiling D, Guoqiang S (2014) Research on forecasting public opinion of public crisis. Intell Bureau. https://doi.org/10.13833 /j.cnki.is.2014.04.011

Zhixue L, Linlin Z (2009) Global stability of an SEIR epidemic model with vertical transmission and saturating contact rate. Chaos Solitons. https://doi.org/10.1016/j.chaos.2007.08.035 